\title{
Dual-Specificity Phosphatases and Kidney Diseases
}

\author{
Haiyang Li Jiachuan Xiong Yu Du Yinghui Huang Jinghong Zhao
}

Department of Nephrology, The Key Laboratory for the Prevention and Treatment of Chronic Kidney Disease of Chongqing, Chongqing Clinical Research Center of Kidney and Urology Diseases, Xinqiao Hospital, Army Medical University (Third Military Medical University), Chongqing, PR China

\section{Keywords}

Dual-specificity phosphatases - Dephosphorylate .

Mitogen-activated protein kinases $\cdot$ Kidney diseases

\section{Abstract}

Background: Dual-specificity phosphatases (DUSPs) belong to the family of protein tyrosine phosphatases, which can dephosphorylate both serine/threonine and tyrosine residues. During the past decades, DUSPs have been implicated in various physiological and pathological activities. Besides mitogen-activated protein kinases (MAPKs) as the main substrates, other protein and nonprotein substrates can also be dephosphorylated by DUSPs. Aberrant regulations of DUSPs have been found in various diseases such as cancer, neurological disorders, and kidney diseases, suggesting the involvement of DUSPs in the pathogenesis of diseases. Summary: In this review, we summarize the general characteristics of DUSPs and the research progress made in the field of kidney diseases, including diabetic nephropathy, hypertensive nephropathy, chronic kidney disease, acute kidney injury, and lupus nephritis. As the main biochemical function of DUSPs is to dephosphorylate MAPKs activity, decreased DUSPs are found in kidney disease models, whereas forced

karger@karger.com www.karger.com/kdd

Karger"
(C) 2021 The Author(s)

Published by S. Karger AG, Basel

This is an Open Access article licensed under the Creative Commons Attribution-NonCommercial-4.0 International License (CC BY-NC) (http://www.karger.com/Services/OpenAccessLicense), applicable to the online version of the article only. Usage and distribution for commercial purposes requires written permission.
DUSPs expression reverses the disease presentation, which was proved by using transgenic or gene knockout model. Key Messages: Mounting evidence demonstrates that DUSPs have essential physiological and pathological functions in kidney disease. Fully understanding the functions and mechanisms of DUSPs in kidney disease contributes to their clinical application in translation medicine.

(c) 2021 The Author(s)

Published by S. Karger AG, Basel

\section{Introduction}

As an important means of post-translational modifications, reversible protein phosphorylation can regulate biological activity and participate in various physiological and pathological processes in eukaryotes. The cellular homeostasis of protein phosphorylation is maintained by certain protein kinases and protein phosphatases, depending on the intracellular and extracellular environments. Eukaryotes have various protein kinases, whose functions are to phosphorylate a protein substrate at specific amino acid residue. One of the best-known kinases

Haiyang Li and Jiachuan Xiong contributed equally to this work.
Correspondence to:

Yinghui Huang, ikkyhuang@163.com Jinghong Zhao, zhaojh@tmmu.edu.cn 
Fig. 1. Schematic representations of the structure of DUSPs proteins. Typical and atypical DUSPs are divided by their structural discrepancy. The gene expression levels of DUSPs are indicated by the colored names, according to the data from Human Protein Atlas. Red, high expression; pink, moderate expression; lime-green, low expression; green, no expression. DUSPs, dual-specificity phosphatases.

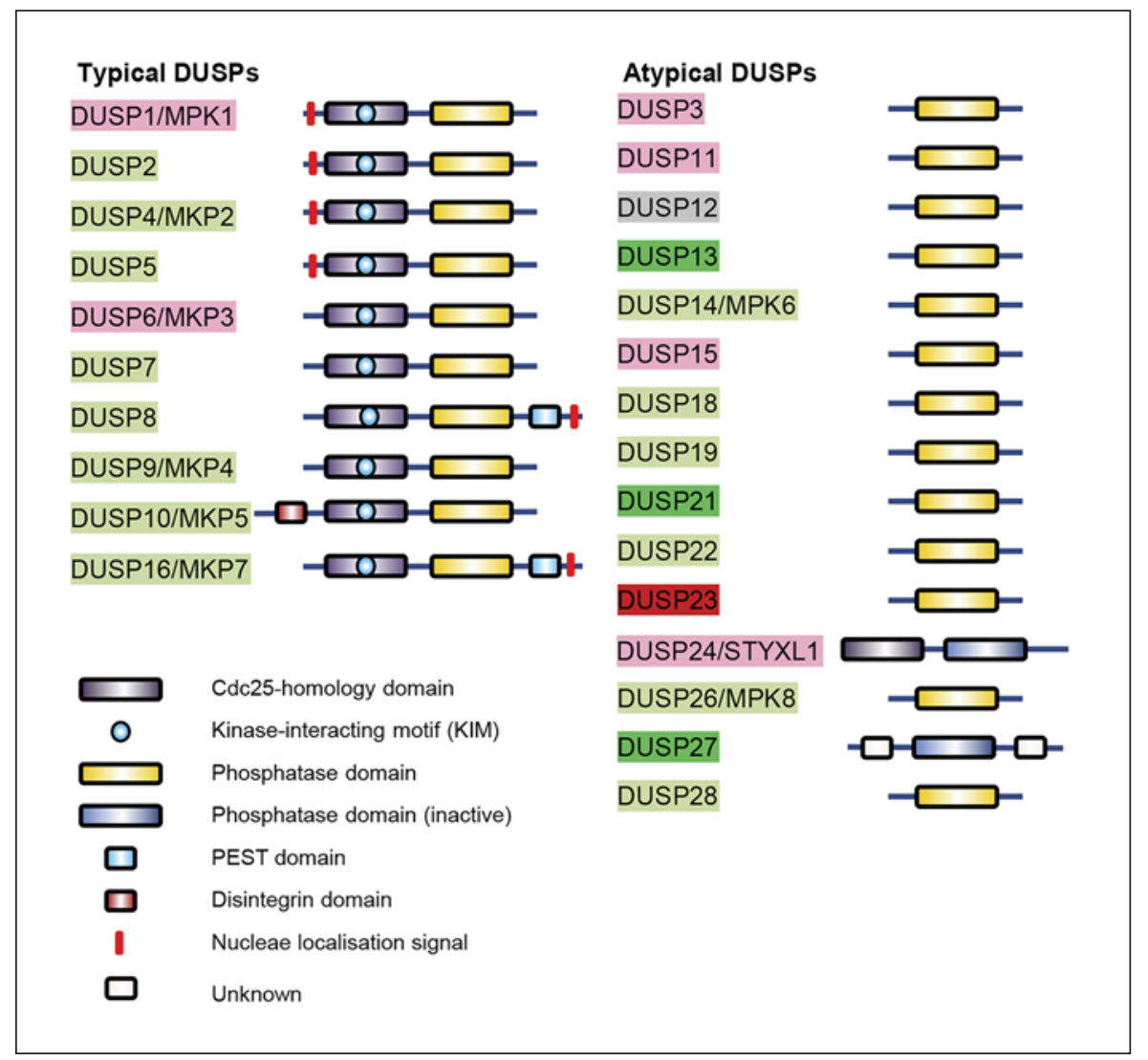

is the mitogen-activated protein kinase (MAPK) cascade, consisting of a linear array of 3 kinases. Besides, numerous protein phosphatases counteract with kinases. Based on the structural similarity and their substrate, protein phosphatases are classified as Ser/Thr phosphatases like PP2A and protein Tyr phosphatase (PTPs) like PTP-SL [1]. Among the PTPs, dual-specificity phosphatases (DUSPs) attract researchers' attention and their roles and mechanisms in various disease models have been extensively explored [2].

DUSPs are also known as MAPK-specific phosphatases (MKPs), with the first member DUSP1/MKP1 identified in 1992 [3]. As indicated by their name, DUSPs possess the biological activity of both Ser/Thr phosphatases and Tyr phosphatase. However, their catalytic activity of Tyr phosphatase is much stronger than that of Ser/Thr phosphatases. Therefore, DUSPs are included in the superfamily of PTPs. In recent years, with the progress made in DUSPs, the strict regulation of reversible phosphorylation in cells has been further recognized, which provides new ideas for the occurrence, development and prevention strategies of some diseases such as cancer, dis- orders of the immune system and neurological disorders $[4,5]$. In this minireview, we will focus on the functions of DUSPs in kidney disease.

\section{Classification and Functions of DUSPs}

Structurally, the catalytic domain of DUSPs contains a conservative motif of HCxxGxxR [6]. Based on the structure, its catalytic activity and substrates, DUSPs are divided into the following subgroups: (i) MAPKs; (ii) atypical DUSPs; (iii) phosphatase and tensin homolog protein phosphatases; (iv) cell division cycle 14 phosphatases (CDC14s); (v) slingshot protein phosphatases, and (vi) phosphatases of the regenerating liver. Among these subgroups, members in the first 2 subgroups have the function of phosphatase with dual specificity for tyrosine and threonine. Therefore, these members are taken as the traditional DUSPs (Fig. 1; Table 1) and will be discussed in the following section.

\section{Mitogen-Activated Protein Kinase}

MAPK pathways regulate many cellular processes, including cell proliferation, differentiation, migration, sur- 


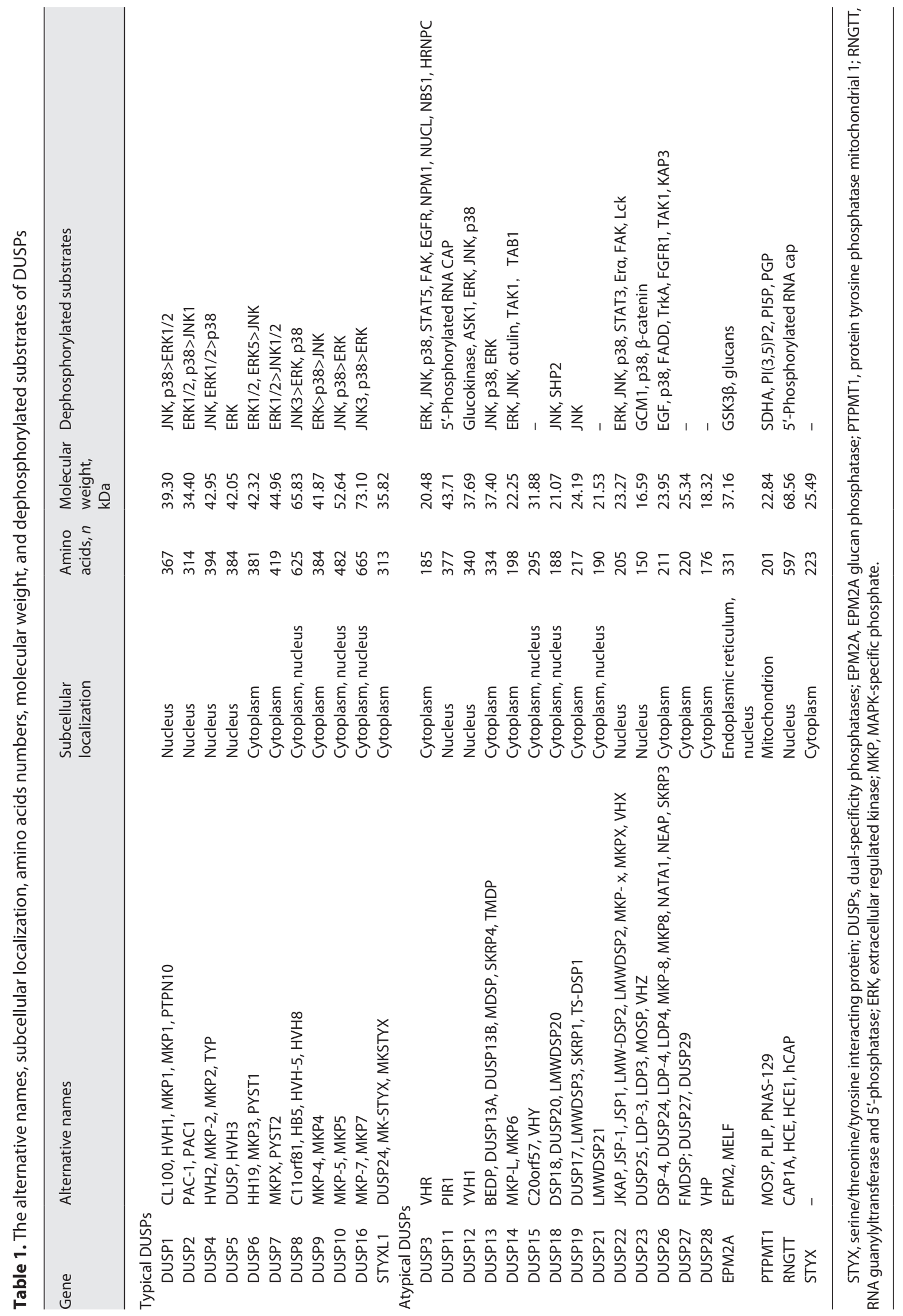


vival, or apoptosis. Aberrant MAPK signaling is involved in many human diseases $[7,8]$. MAPK pathways can be activated by a variety of stimuli, kinases, and other enzymes. The MAPK signaling contains a 3-tiered cascade, including a MAP kinase kinase kinase), a MAP kinase kinase, and the MAPK. There are 3 major MAPK pathways, extracellular regulated kinases 1 and 2 (ERK1/2), c-Jun-N-terminal kinases (JNKs), and p38. MAPKs pathways are negatively regulated by MKPs, which can dephosphorylate the active MAPK substrates. So far, due to the uncertainty of the efficacy or the existence of potential adverse effects, no MAPK inhibitors or relative drugs have been clinically tested for the treatment of human diseases. Clinical treatment targeting the upstream molecules of the MAPK pathway is much better than that targeting the downstream molecules, according to the current findings of the MAPK inhibitors. Therefore, MPKs might be applied in translation medicine.

There are 10 members in the MPKs family. Each member is composed of the MAPK-binding (MKB) domain in the N-terminal end and the conserved DUSP catalytic domain in the $\mathrm{C}$-terminal end. The kinase-interaction motif of the MKB domain determines the enzymatic specificity through docking interaction with MAPK [9]. Of note, both phosphorylated and unphosphorylated MAPK can bind with MPKs [10]. After binding with MAPK, the conformation of MPKs protein is altered and the catalytic activity of MKPs is thus increased.

According to their cellular distribution, the MPKs members can be divided into 3 subgroups: (1) nuclear MPKs, including DUSP1/MKP1, DUSP2, DUSP4/MKP2, and DUSP5; (2) cytosolic MPKs, including DUSP6/ MKP3, DUSP7, and DUSP9/MKP4; and (3) both nuclear and cytosolic MPKs, including DUSP8, DUSP10/MKP5, and DUSP16/MKP7. Although these MPKs were reported to dephosphorylate MAPKs, the substrates of MPKs are not precisely defined. Substrate preferences of MPKs are associated with the nature of the binding site and cell type, as well as the scaffold proteins of MAPKs [11].

\section{Atypical DUSPs}

Atypical DUSPs are identified by their structural similarity with that of MPKs. This category of proteins is usually smaller than MPKs. Atypical DUSPs contain the DSP catalytic domain, but lack the $\mathrm{N}$-terminal MKB domain. In addition, some members also contain the $\mathrm{CH} 2$ domain, the carbohydrate-binding domain, and an Arginine-rich or Proline-rich region. In mammalian tissues, there are about 20 members, DUSP3, DUSP11, DUSP12, DUSP13, DUSP14, DUSP15, DUSP18, DUSP19, DUSP21,
DUSP22, DUSP23, DUSP26, DUSP27, DUSP28, EPM2A glucan phosphatase, Protein tyrosine phosphatase mitochondrial 1, RNA guanylyltransferase and $5^{\prime}$-phosphatase, and Serine/threonine/tyrosine interacting protein. However, these atypical DUSPs are derived from a common ancestor as revealed by phylogeny analysis [12].

Because atypical DUSPs lack the specific MKB domain of MKPs, their exact substrates are still uncertain. Among these atypical DUSPs members, DUSP14/MKP6 and DUSP26/MKP8 are reported to bind with MAPKs and regulate the signaling pathway. Besides MAKPs, atypical DUSPs can function on other phosphorylated protein substrates. Moreover, they can dephosphorylate nonprotein substrates such as RNA or lipids. For example, DUSP11 has intrinsic phosphatase activity, and its activity of mRNA triphosphatase is greater than that of protein phosphatase [13]. In addition, atypical DUSPs can also act as scaffold proteins to facilitate the interaction of signaling proteins [14]. Therefore, atypical DUSPs have extensive substrate specificity and versatile physiological functions.

\section{Expression and Regulation of DUSPs in Kidney}

The main cellular components of the kidney include tubular epithelial cells, mesangial cells, podocytes, and interstitial cells, which are most often studied in renal disease models. In the glomeruli, the main constituents are mesangial cells, which account for approximately 30$40 \%$ of the total cells [15]. DUSPs are expressed in various types of cells of the kidney at different levels. Renal tubular epithelial cells are the most abundant cell type of kidney, and these genes, including DUSP1/MKP1, DUSP4/ MKP2, and DUSP7, were reported to be expressed in tubular cells in vivo under various kidney disease models or in immortalized cell lines like HK-2. Moreover, DUSP1/ MKP1 and DUSP10/MKP5 were expressed in renal mesangial cells, while DUSP4/MKP2 and DUSP6/MKP3 were found in podocytes $[16,17]$. In addition, vascular cells in the kidney, like vascular smooth muscle cells (VSMCs), can also express DUSPs. DUSP1/MKP1 and DUSP5 were reported to be expressed in VSMCs and might have a role in hypertension-relative kidney disease. Of note, resident immunological cells in the kidney such as T-cell, B-cell, and mononuclear cells can express DUSPs $[18,19]$. Although the expression of partial DUSPs members was revealed and compared under those disease models, the gene expression of most members was unknown. In addition, the exact expression levels of DUSPs are not clear. Recently, single-cell sequencing has provided the possibility to compare the expression level of an 


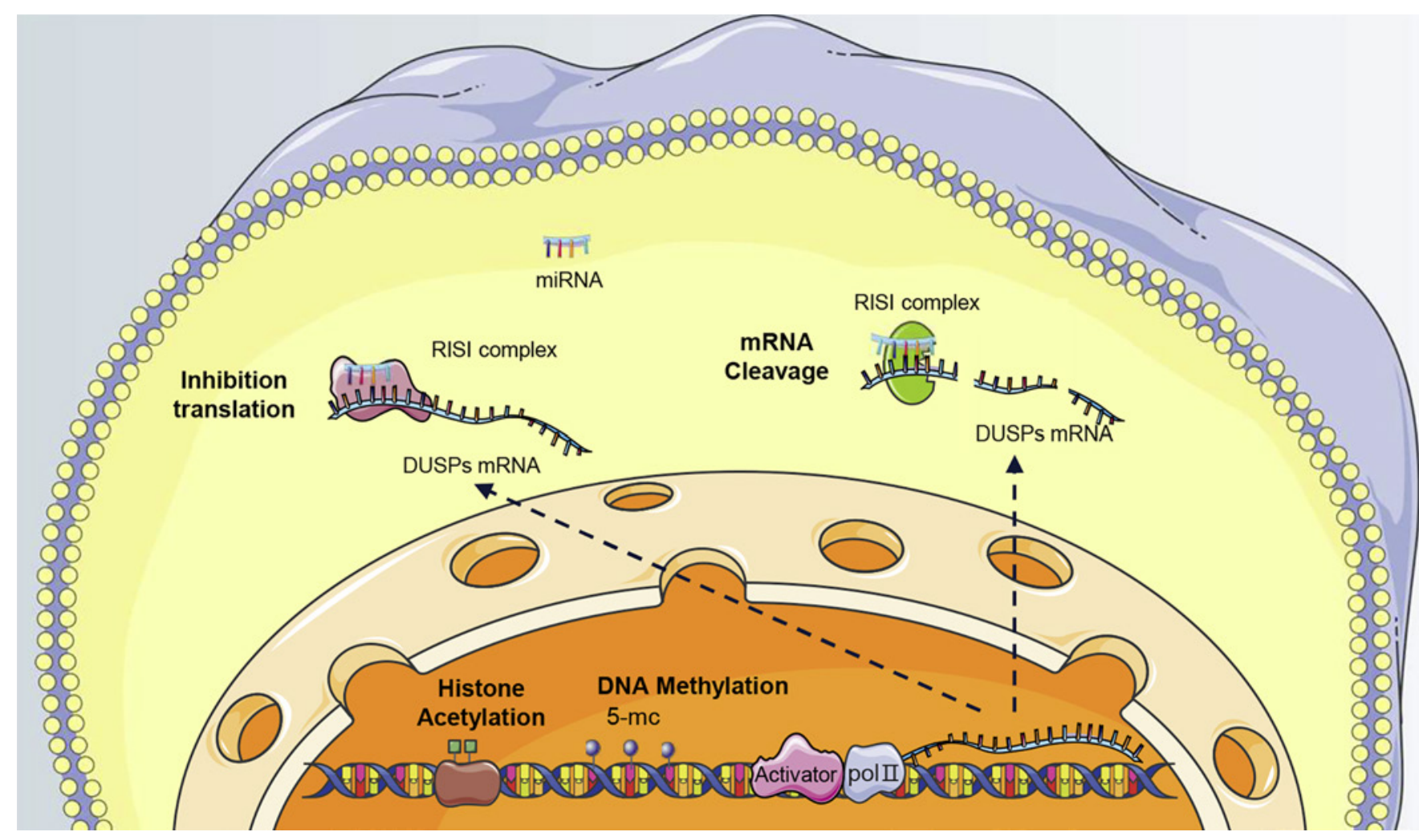

Fig. 2. Gene expression of DUSPs. Transcription of DUSPs can be regulated by all epigenetic means including DNA methylation, histone modification, and noncoding RNAs. DUSPs, dual-specificity phosphatases.

individual gene. Based on the data from Human Protein Atlas (www.proteinatlas.org), the expression level of DUSPs can be arbitrarily divided into 4 groups. DUSP23 belongs to the high expression group, while DUSP1/ MKP1, DUSP6/MKP3, DUSP3, DUSP11, DUSP15, DUSP24/Serine/threonine/tyrosine interacting proteinL1, and Protein tyrosine phosphatase mitochondrial 1 belong to the moderate expression group. DUSP13, DUSP21, and DUSP27 are not expressed in the kidney, while the left members belong to the low expression group. Therefore, the gene expression of DUSPs needs further investigation, especially in disease models.

Gene expression and phosphatase activity of DUSPs can be regulated by means of gene transcription, translational regulation, protein modification, or protein stability. Aberrant expression of DUSPs was found in various models in which deregulation of DUSPs can occur at the transcriptional level. For instance, both protein and mRNA levels of DUSP1/MKP1 were downregulated in diabetic renal tissues [20]. Similarly, diabetes and high glucose exposure decreased DUSP4/MKP2 expression at the transcriptional level in cultured podocytes and glomeruli, which led to enhanced p38 and JNK activity [16].

As expected, besides other vital transcriptional factors, transcription of DUSPs can also be regulated by epigenetic means such as DNA methylation (Fig. 2). Currently, DNA methylation generally represents a repressive epigenetic mark that silences gene expression. Hypermethylation of promoter regions of DUSPs leads to decreased expression, which has been proved in many cell types. Analyzed by the methylation-specific PCR, a significant decrease of DUSP1/MKP1 expression in both breast cancer cell lines and invasive breast tumors was found associated with DNA methylation, and the promoter methylation of DUSP1/MKP1 might be a potential breast cancer biomarker for breast malignancy [21]. Similarly, Tögel et al. [22] found that DUSP5 was methylated in CIMP-high colorectal cancer, although this epigenetic change alone could not account for reduced DUSP5 expression in cancer cells. Besides DNA methylation, histone modification is also involved in the regulation of DUSPs. The modification includes histone acetylation, methylation, and 
phosphorylation, and the effects on gene expression depend on the nature of histone modification. Recently, Hofmann et al. [23] proved that CREMa could regulate DUSP 4 expression by inducing histone acetylation at the DUSP4 promoter via p300 in effector T cells. In kidney disease models, Coit et al. [19] analyzed the genome-wide DNA methylation changes in naïve $\mathrm{CD} 4^{+} \mathrm{T}$ cells among Lupus patients with or without renal involvement and health control and found that DUSP5 was hypomethylated. DUSP5 demethylation might contribute to a defective ERK signaling pathway in lupus T cells. Up-to-date studies regarding the epigenetic regulation of DUSPs in kidney disease are relatively insufficient. More attention should be paid in this research area.

In addition, the expression of DUSPs can be regulated at post-transcriptional or translational levels. Noncoding RNAs have an important role in the regulation of DUSPs. These RNAs, such as miRNAs and long non-coding RNAs (lncRNAs), can work directly or indirectly. Usually, miRNAs can function by directly targeting DUSPs mRNA, for example, miR-107 was verified to target the $3^{\prime}$ UTR of DUSP7 in endothelial cells [24], and DUSP4 was a downstream target of miR-122-5p [25]. These miRNAs lead to a translational decrease of DUSPs proteins. Unlike miRNAs, lncRNAs can function indirectly, either via binding with miRNAs or recruiting other proteins. Recently, IncRNA AZIN1 was found to downregulate miR$513 \mathrm{~b}-5 \mathrm{p}$ via sponging it, which in turn targeted DUSP11 in tumor tissues [26]. More interestingly, another study shows that lncRNA CASC9 could recruit histone methyltransferase EZH2 to be epigenetic regulate DUSP1/MKP1 expression in non-small cell lung cancer [27]. These studies revealed the translational regulation of DUSPs.

Finally, regulation of DUSPs can also occur at the protein level. DUSP proteins can be acetylated, phosphorated, methylated, or ubiquitinated. These modifications can either affect their biological activity or protein stability. The detailed regulation of DUSPs at the protein level can be found in other reviews [28].

\section{DUSPs in Kidney Disease}

In this section, we will discuss the functions of DUSPs in kidney disease models, including diabetic nephropathy (DN), hypertensive nephropathy, chronic kidney disease (CKD), acute kidney injury (AKI), and lupus nephritis (LN), focusing on the roles of DUSPs (Table 2). As the main biochemical function of DUSPs is to dephosphorylate MAPKs activity, decreased DUSPs are found in kidney disease models, whereas forced DUSPs expression reverses the disease presentation. At the molecular level, most studies focus on the regulation of MAPKs by DUSPs (Fig. 3). Recent progress has shed light on the clinical translation based on DUSPs for such kidney diseases.

\section{Diabetic Nephrology}

$\mathrm{DN}$, a leading cause of end-stage renal disease, is a common microvascular complication of diabetes mellitus. Due to the complex metabolic disorder, the clinical treatments of DN are much more troublesome than those for other kidney diseases, and few treatment options are available to prevent the progressive loss of renal function. The etiology of DN is more complicated than expected. Besides the genetic factors, high glucose, glycosylation products, renal hemodynamics, cytokines, and other factors are associated with DN, and the deregulation of signaling pathways happens. The activation of MAPKs signaling pathways, especially p38 and JNK MAPK, is closely related to the occurrence and development of $\mathrm{DN}$ [29-33]. A growing body of studies has demonstrated that p38 MAPK is hyper-activated in the glomeruli of DN mouse or DN patients, while the inhibition of the p38 MAPK pathway has a beneficial effect on the development of DN by inhibiting the increase in fibronectin expression and apoptosis. Although the detailed molecular mechanisms underlying why MAPKs are activated in DN models have not been completely elucidated, DUSPs, as the specific inhibitors of MAPKs, are confirmed to have an important role in the pathogenesis of $\mathrm{DN}$ mediated by MAPKs, evidenced by the following findings.

First, deregulation of DUSPs has been observed in various DN models. DUSP1/MKP1 is the first identified and most widely studied member of the DUSPs family. It is expressed in a variety of tissues and cells, and participates in a variety of biological events such as cell death, cancer, inflammation and metabolic diseases. Decreased expression of DUSP1/MKP1 was detected in the kidney of STZmediated diabetic mice, which contributed to the development of diabetes [20]. Moreover, both in the mesangial cells and tubular epithelial cells, treatment of high glucose led to significant decrease of DUSP1/MKP1 expression, which was consistent with the findings in vivo. Similarly, a reduction of DUSP4/MKP2, DUSP6/MKP3, and DUSP10/MKP5 was also observed in the diabetic kidney of STZ-treated mice [34]. As for atypical DUSPs, only DUSP26 was investigated in DN mouse. Huang et al. [35] found that DUSP26 expression was downregulated in kidney tissues of patients and mouse models with DN. The biomarkers of DN in DUSP26-KO mice were worse than that in WT mice treated with streptozotocin. All these studies clearly show that DUSPs are downregulated 


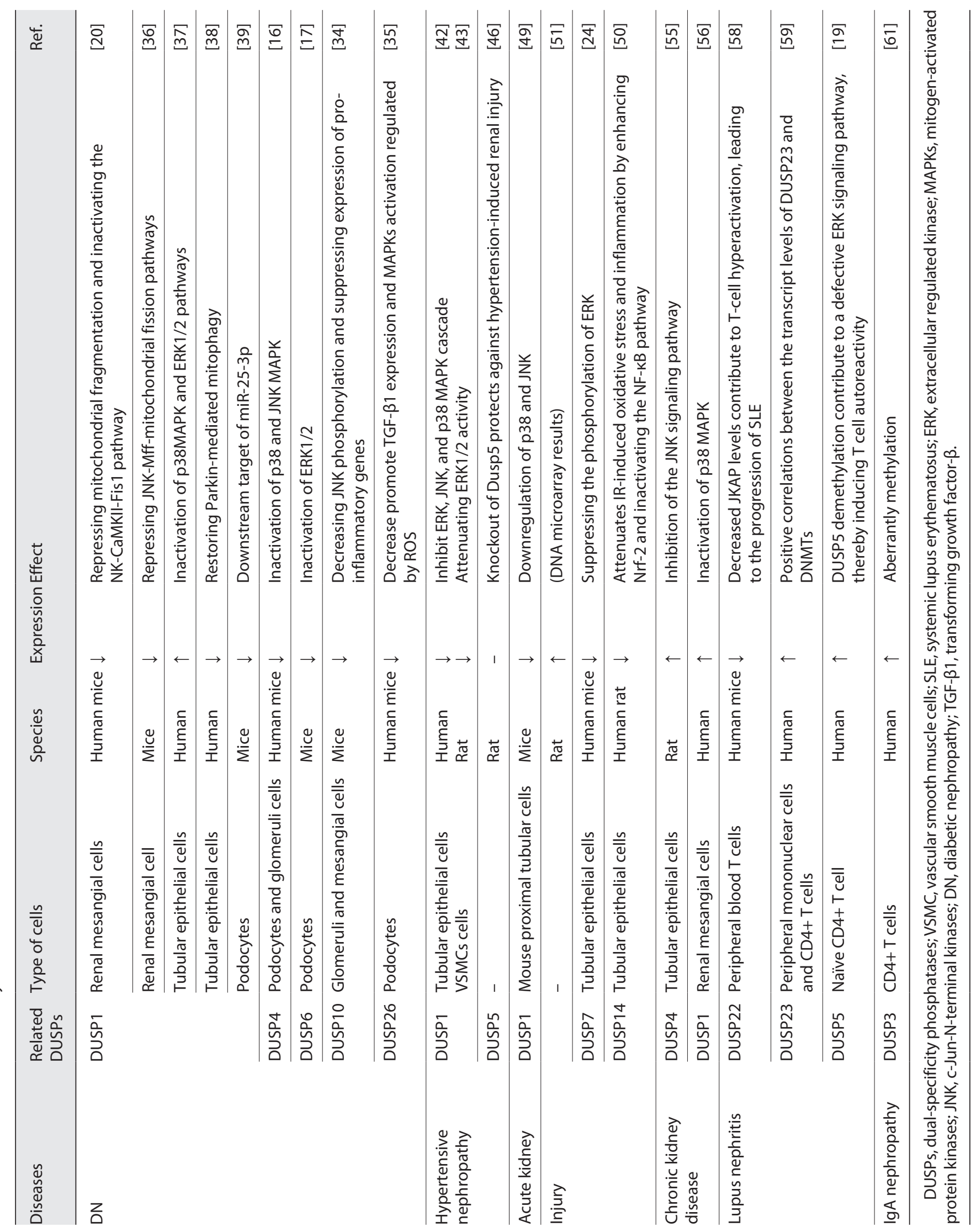




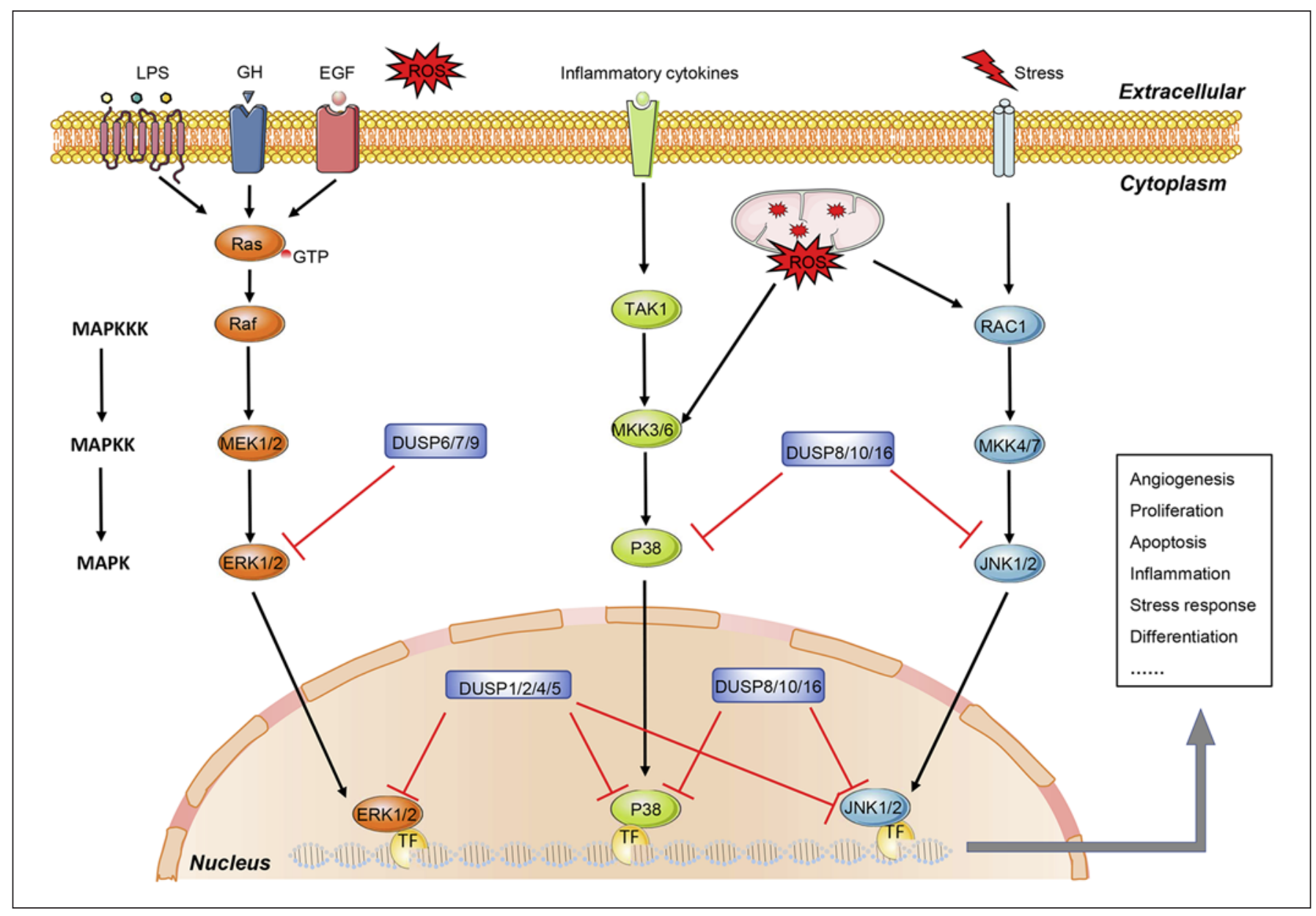

Fig. 3. Molecular mechanisms of the main DUSPs that control MAPKs in the kidney. DUSP, dual-specificity phosphatases; MAPKs, mitogen-activated protein kinases.

in DN models, suggesting a causative pathological role in DN.

Second, functional analysis of DUSPs shows that decreased DUSPs contribute to the activation of MAPKs in $\mathrm{DN}$. As the activation of MAPKs is common in DN, it is not surprising to find decreased DUSPs expression. However, their substrates might be different. In renal mesangial cells, DUSP1/MKP1 preferred JNK pathway, which was the upstream activator for Mff phosphorylation [36]. In tubular epithelial cells, overexpression of DUSP1/ MKP1 ameliorated the HG-induced expression levels of collagen I, collagen IV, and fibronectin via inactivation of p38 and ERK1/2 pathways $[37,38]$. However, in another study, the author demonstrated that M2 macrophage-derived exosomal miR-25-3p could improve HG-induced podocyte injury through activating autophagy, and DUSP1/MKP1 was proved to be a downstream target and mediated the inhibition of exosomal miR-25-3p to HGinduced podocytes injury [39]. The different roles of DUSP1/MKP1 might be related to the cell type. In cultured podocytes and glomeruli, diabetes-induced DUSP4 reduction enhanced p38 and JNK activity, while overexpression of DUSP 4 prevented the activation of p38 and JNK. However, DUSP26 inhibition enhanced the activation of all 3 main types of MAPKs in podocytes [35]. These studies showed the substrate preference of individual DUSPs members.

Third, genetic alterations of DUSPs can regulate the progress of DN. Transgenic and gene knockout (KO) mouse of DUSPs has been generated to observe the effects of DUSPs in DN. Currently, gene deletion of most members of DUSPs family is available for functional analysis. Mice with global deletion of DUSPs are fertile, for example, DUSP1/MKP1, DUSP4, DUSP22, and 
DUSP26-KO mice have been tested in DN models. Consistent with the findings that their expression was downregulated, $\mathrm{KO}$ mice showed an accelerated DN progression, as proved by the promoted development of albuminuria and glomerulosclerosis. Contrarily, transgenic mice showed an opposite effect. For instance, DUSP1/ MKP1 overexpression could reduce high glucose-induced glomerular apoptosis in DUSP1/MKP1 transgenic mice [36]. However, the studies involved with DN patients are very limited.

In summary of this section, DUSPs play an important pathological role in DN. The downregulation of DUSPs contributes to the activation of MAPKs. As overexpression of DUSPs is beneficial for DN animals, pharmacological activation of DUSPs might be an option for translational medicine.

\section{Hypertensive Nephropathy}

The kidney can participate in the regulation of blood pressure (BP) through the secretion of renin [40]. Therefore, the kidney is not only one of the important target organs of hypertension damage, but also an organ leading to hypertension. Hypertensive nephropathy is the second leading cause of end-stage renal disease after DN. The prevalence of hypertensive nephropathy is increasing, and $>20 \%$ of hypertensive patients develop renal dysfunction. Renovascular hypertension is a kind of syndrome, which refers to the decrease of renal perfusion due to the injury of renal vessels and results in arterial hypertension. High (BP) can affect each renal compartment, including vessels, glomeruli, and podocyte damage. In recent years, several evidences showed that high $\mathrm{BP}$ also injures tubular cells, such as tubulointerstitial fibrosis and epithelial-to-mesenchymal transition (EMT) [41]. However, the clinical treatments of hypertensive nephropathy are relatively limited, with the aim to protect the residual nephrons and to delay the progression of renal damage using antihypertensive drugs. Numerous studies have investigated the renin-angiotensin system, oxidative stress, endothelial dysfunction, inflammatory response, and genetic factors to explore the pathogenesis of hypertensive nephropathy. However, the detailed pathogenesis of hypertensive nephropathy remains poorly understood.

Recently, several studies have shown that DUSPs are also involved in the pathogenesis of hypertensive nephropathy. By analyzing the microarray data of GSE99325 and GSE32591 from Gene Expression Omnibus, Chen et al. found that a total of 267 genes, including DUSP1/ MKP1 were identified as differentially expressed genes associated with hypertensive nephropathy. And the downregulation of DUSP1/MKP1 was further confirmed in a cell model of HK-2 treated with angiotensin II [42]. In VSMCs cells, Angiotensin (Ang)-(1-7) counter-regulated the actions evoked by Ang II overproduction mainly by modulating MKP1 activity [43]. This finding suggested that DUSP1/MKP1 may contribute to the progression of hypertensive nephropathy. DUSP5 belongs to nuclear MKPs, which can be induced by heat shock protein and growth factors in mammalian cells. ERK1/2 is the primary substrate of DUSP5 and can be dephosphorylated by its phosphatase activity, while a decrease of DUSP5, in turn, can enhance the amplitude and duration of ERK signal transduction [44, 45]. On the contrary, ERK can maintain the stability of DUSP5 by inhibiting the ubiquitination of DUSP5, which is independent of ERK kinase activity but depends on the interaction of ERK-DUSP5. By using DUSP5 knockout mice, Zhang et al. [46] found that hypertension-induced renal injury, including renal blood flow, proteinuria, glomerular injury, and renal fibrosis, was markedly ameliorated in hypertensive $\mathrm{KO}$ rats. Of note, most DUSPs KO animals showed a deteriorated phenotype in the disease model, but $\mathrm{KO}$ of DUSP5 had a renoprotective effect. This might be related to the cell type and its substrate preference of DUSPs members. In VSMCs, activated ERK1/2 and PKC promote calcium influx and facilitate vasoconstriction [47]. In DUSP5 KO mice, myogenic reactivity of the afferent arteriole and preglomerular arteries were enhanced, and the autoregulation of renal blood flow was improved following the induction of DOCA-salt hypertension, which was companied with the reduced expression of MCP-1 and decreased infiltration of macrophages, as well as the decreased expression of transforming growth factor- $\beta$ (TGF- $\beta 1$ ), MMP2, and MMP9 in the kidney. Therefore, KO of DUSP5 protected against hypertension-induced renal injury. The roles and mechanisms of other DUSPs members in hypertensive nephropathy need further investigation.

\section{Acute Kidney Injury}

AKI is a common and critical clinical syndrome characterized by a rapid decline in renal function. Due to its clinical implications like organ failure and poor outcomes, AKI has received a great deal of attention in recent years. AKI can result from ischemic and pharmacological insults. The ultimate renal injury is determined not only by the direct effects of insults but also by the maladaptive responses of renal tissues. During the past decades, large efforts have been made to elucidate the roles of inflamma- 
tion and cytokines, and many genes or vital players involved in AKI have been identified. However, the pathophysiological mechanism of AKI has not been fully elucidated. Activation of MAPKs was found to be related with AKI-induced cell apoptosis, which indicates that MAPK inhibition contributes to the mitigation of AKI. For example, cisplatin increased renal injury through upregulation of p38 and JNK [48]. Therefore, DUSPs are thought to have a role in AKI, as they are the specific phosphatases of MAPKs.

In the cisplatin-induced mouse model, DUSP1/MKP1 expression was downregulated by cisplatin, which might be a new possible mechanism of cisplatin-induced phosphorylation of MAPK (JNK and p38) in renal injury [49]. The authors thought that regulation of MKP1 expression could be a new method to ameliorate cisplatin-induced renal injury. Similarly, decreased DUSP7 and increased phosphorylated ERK were observed in the endothelial cells of septic AKI mouse, and treatment with antisense oligonucleotides of miR-107 recovered the expression of DUSP7, suppressed the phosphorylation of ERK, and decreased the secretion of TNF- $\alpha$ in septic AKI mice [24]. As for atypical DUSPs, Xu et al. [50] have shown that DUSP14 expression was decreased in the ischemia-reperfusion (IR) rat model, and upregulated DUSP14 attenuates IR-induced oxidative stress and inflammation by enhancing Nrf-2 and inactivating the NF- $\kappa B$ pathway. However, unexpected upregulation of DUSP1/MKP1 was observed by using DNA microarray in a rat model of AKI, which contradicted other findings [51]. Up to date, the roles of other DUSPs members in AKI have not been explored. Anyway, these studies provide novel clues on the mechanism of DUSPs in AKI and the potential treatment of AKI.

\section{Chronic Kidney Disease}

With the growing morbidity and mortality, CKD is becoming a global public health challenge that poses a heavy burden on patients and society. Renal inflammation and interstitial fibrosis are the universal pathological manifestations in the progression of CKD. At the molecular level, many growth factors and cytokines participate in the process, leading to the differentiation of renal interstitial fibroblasts into the activated myofibroblast phenotype. In addition, chronic inflammation, oxidative stress, and aberrant metabolism contribute to CKD. Notably, EMT is one of the mechanisms related to renal interstitial fibrosis, and TGF- $\beta 1$ plays a critical role in the progression of EMT [52].
All 3 MAP kinase pathways are activated in CKD patients, while pharmacologic or genetic strategies to block MAPKs can suppress renal fibrosis in a variety of animal models $[53,54]$. As negative regulators of MAPKs, DUSPs should play a role in CKD. Li et al. [55] found that the expression of DUSP4/MKP2 was upregulated in a unilateral ureter obstruction rat model and in the cell model of NRK-52E cells treated with TGF- $\beta 1$. Overexpression of DUSP 4 attenuated TGF- $\beta 1$ induced EMT via the inhibition of the JNK signaling pathway, while DUSP 4 knockdown promoted TGF- $\beta 1$ induced EMT by enhancing JNK phosphorylation. This finding demonstrated that DUSP4, as a TGF- $\beta 1$ induced negative regulator, regulated EMT in renal tubular epithelial cells via a JNK-dependent pathway. In glomerular mesangial cells, treatment with connective tissue growth factor resulted in the activation of these cells, which could produce ECM (extracellular matrix). Wahab et al. [56] found that connective tissue growth factor promoted activated mesangial cell survival via upregulation of DUSP1/MKP1, suggesting a pro-fibrosis role in the mesangial cell. However, CKD is a complicated pathophysiological change involving in apoptosis, inflammation, and TGF- $\beta 1 /$ Smad pathway, etc. Thus, the roles and mechanisms of DUSPs members in CKD need further study.

\section{Lupus Nephritis}

$\mathrm{LN}$ is a common manifestation of systemic lupus erythematosus (SLE) that causes renal injury, and it is the second main reason of secondary glomerulonephritis in China [57]. The pathologic classification of $\mathrm{LN}$ is predominantly based on renal glomerular inflammation. Several studies indicated the involvement of DUSPs in LN. As previously mentioned, aberrant expression of DUSPs can be found in LN patients. Tse-Hua et al. found that DUSP22 protein level in peripheral blood $\mathrm{T}$ cells was significantly decreased in SLE patients and was inversely correlated with SLE disease activity index and antidsDNA antibody levels. In addition, DUSP22 downregulation in T cells was highly correlated with daily urinary protein amounts and with poor renal outcomes in LN patients [58]. Therefore, the authors concluded that DUSP22 downregulation in T cells was a novel diagnostic and prognostic biomarker for SLE nephritis. On the contrary, DUSP23 mRNA level was found to be increased in $\mathrm{CD}^{4+} \mathrm{T}$ cells from SLE patients, although no association was found between DUSP23 mRNA expression and the presence of typical serological and clinical parameters associated with SLE [59]. Moreover, DNA methyla- 
tion and histone modification are associated with gene regulation in immune cells $[23,60]$. This is like that in IgA nephropathy patients, whose DNA in $\mathrm{CD}^{4+} \mathrm{T}$-cells were found to be aberrantly methylated [61]. LN can be defined as a kind of autoimmune disease. Increasing studies showed that DUSPs are involved in either autoimmune diseases (e.g., DUSP2, DUSP7, DUSP10, and DUSP12) or T-cell activation (e.g., DUSP1/MKP1, DUSP5, and DUSP14) [62-64]. Therefore, more DUSPs members will be found to play a role in the pathogenesis of LN.

\section{Conclusions}

As a type of tyrosine, serine/threonine bispecific phosphatase, DUSPs have attracted much attention. Previous studies on DUSPs mainly focused on cancer research. Recently, DUSPs are shown to be associated with many important cellular processes. Mounting evidence demonstrates that DUSPs have essential physiological and pathological functions not only under the normal condition, but also in kidney diseases. DUSPs are critical regulators of DN, AKI, hypertensive nephropathy, and renal fibrosis. However, little is known regarding the gene expres- sion and precise functions of DUSPs in the human kidney, which needs to be elucidated in the future. Nevertheless, understanding the roles and regulatory mechanisms of DUSPs members in kidney disease will help to provide new ideas for the prevention and treatment of kidney disease. The DUSPs family will be a new target in the field of kidney disease.

\section{Conflict of Interest Statement}

The authors have no conflicts of interest to disclose. J.H.Z. is now the Managing Editor/Associate Editor of the journal.

\section{Funding Sources}

This work was financially supported by the National Natural Science Foundation of China (81800616).

\section{Author Contributions}

H.Y.L. and J.C.X. wrote the draft of the review; Y.D., Y.H.H., and J.H.Z. revised the review; J.H.Z. designed and approved this work.

\section{References}

1 Salazar C, Höfer T. Multisite protein phosphorylation--from molecular mechanisms to kinetic models. FEBS J. 2009 Jun;276(12): 3177-98.

2 Owens DM, Keyse SM. Differential regulation of MAP kinase signalling by dual-specificity protein phosphatases. Oncogene. 2007 May 14;26(22):3203-13.

3 Charles $\mathrm{CH}$, Abler AS, Lau LF. cDNA sequence of a growth factor-inducible immediate early gene and characterization of its encoded protein. Oncogene. 1992 Jan;7(1):18790.

4 An N, Bassil K, Al Jowf GI, Steinbusch HWM, Rothermel M, de Nijs L, et al. Dual-specificity phosphatases in mental and neurological disorders. Prog Neurobiol. 2021 Mar; 198: 101906.

5 Mutlak M, Kehat I. Dual specific phosphatases (DUSPs) in cardiac hypertrophy and failure. Cell Signal. 2021 Aug;84:110033.

6 Alonso A, Pulido R. The extended human PTPome: a growing tyrosine phosphatase family. FEBS J. 2016 Apr;283(8):1404-29.

7 Kim EK, Choi EJ. Compromised MAPK signaling in human diseases: an update. Arch Toxicol. 2015 Jun;89(6):867-82.

DUSPs and Kidney Diseases
8 Kurtzeborn K, Kwon HN, Kuure S. MAPK/ ERK signaling in regulation of renal differentiation. Int J Mol Sci. 2019 Apr 10;20(7).

9 Kondoh K, Nishida E. Regulation of MAP kinases by MAP kinase phosphatases. Biochim Biophys Acta. 2007 Aug;1773(8):1227-37.

10 Kidger AM, Keyse SM. The regulation of oncogenic Ras/ERK signalling by dual-specificity mitogen activated protein kinase phosphatases (MKPs). Semin Cell Dev Biol. 2016 Feb; 50:125-32.

11 Patterson KI, Brummer T, O'Brien PM, Daly RJ. Dual-specificity phosphatases: critical regulators with diverse cellular targets. Biochem J. 2009 Mar 15;418(3):475-89.

12 Huang CY, Tan TH. DUSPs, to MAP kinases and beyond. Cell Biosci. 2012 Jul 9;2(1): 24.

13 Kincaid RP, Lam VL, Chirayil RP, Randall G, Sullivan CS. RNA triphosphatase DUSP11 enables exonuclease XRN-mediated restriction of hepatitis $\mathrm{C}$ virus. Proc Natl Acad Sci U S A. 2018 Aug 7;115(32):8197-202.

14 Armstrong SP, Caunt CJ, McArdle CA. Gonadotropin-releasing hormone and protein kinase C signaling to ERK: spatiotemporal regulation of ERK by docking domains and dual-specificity phosphatases. Mol Endocrinol. 2009 Apr;23(4):510-9.
15 Abboud HE. Mesangial cell biology. Exp Cell Res. 2012 May 15;318(9):979-85.

16 Denhez B, Rousseau M, Dancosst DA, Lizotte F, Guay A, Auger-Messier M, et al. Diabetesinduced DUSP4 reduction promotes podocyte dysfunction and progression of diabetic nephropathy. Diabetes. 2019 May;68(5): 1026-39.

17 Chen L, Wang Y, Luan H, Ma G, Zhang H, Chen G. DUSP6 protects murine podocytes from high glucose: induced inflammation and apoptosis. Mol Med Rep. 2020 Sep;22(3): 2273-82.

18 Li JP, Yang CY, Chuang HC, Lan JL, Chen DY, Chen YM, et al. The phosphatase JKAP/ DUSP22 inhibits $\mathrm{T}$-cell receptor signalling and autoimmunity by inactivating Lck. Nat Commun. 2014 Apr 9;5:3618.

19 Coit P, Renauer P, Jeffries MA, Merrill JT, McCune WJ, Maksimowicz-McKinnon K, et al. Renal involvement in lupus is characterized by unique DNA methylation changes in naïve CD4+ T cells. J Autoimmun. 2015 Jul; 61:29-35. 
20 Zhang Y, Feng J, Wang Q, Zhao S, Yang S, Tian L, et al. Hyperglycaemia stress-induced renal injury is caused by extensive mitochondrial fragmentation, attenuated MKP1 signalling, and activated JNK-CaMKII-Fis1 biological axis. Cell Physiol Biochem. 2018;51(4): 1778-98.

21 Chen FM, Chang HW, Yang SF, Huang YF, Nien PY, Yeh YT, et al. The mitogen-activated protein kinase phosphatase-1 (MKP-1) gene is a potential methylation biomarker for malignancy of breast cancer. Exp Mol Med. 2012 May 31;44(5):356-62.

22 Tögel L, Nightingale R, Wu R, Chüeh AC, AlObaidi S, Luk I, et al. DUSP5 is methylated in CIMP-high colorectal cancer but is not a major regulator of intestinal cell proliferation and tumorigenesis. Sci Rep. 2018 Jan 29;8(1): 1767.

23 Hofmann SR, Mäbert K, Kapplusch F, Russ S, Northey S, Beresford MW, et al. cAMP response element modulator a induces dual specificity protein phosphatase 4 to promote effector T cells in juvenile-onset lupus. J Immunol. 2019 Dec 1;203(11):2807-16.

24 Wang S, Zhang Z, Wang J, Miao H. MiR-107 induces TNF- $\alpha$ secretion in endothelial cells causing tubular cell injury in patients with septic acute kidney injury. Biochem Biophys Res Commun. 2017 Jan 29;483(1):45-51.

$25 \mathrm{Hu} \mathrm{N}$, Tian Y, Song Y, Zang L. miR1225p suppresses the oncogenesis of PTC by inhibiting DUSP4 expression. Mol Med Rep. 2021 May; 23(5):368.

26 Cai Y, Wu Q, Liu Y, Wang J. AZIN1-AS1, a novel oncogenic LncRNA, promotes the progression of non-small cell lung cancer by regulating MiR-513b-5p and DUSP11. Onco Targets Ther. 2020;13:9667-78.

27 Chen Z, Chen Q, Cheng Z, Gu J, Feng W, Lei $\mathrm{T}$, et al. Long non-coding RNA CASC9 promotes gefitinib resistance in NSCLC by epigenetic repression of DUSP1. Cell Death Dis. 2020 Oct 14;11(10):858.

28 Chen HF, Chuang HC, Tan TH. Regulation of Dual-Specificity Phosphatase (DUSP) ubiquitination and protein stability. Int J Mol Sci. 2019 May 30;20(11):2668.

29 Makkinje A, Quinn DA, Chen A, Cadilla CL, Force T, Bonventre JV, et al. Gene 33/Mig-6, a transcriptionally inducible adapter protein that binds GTP-Cdc42 and activates SAPK/ JNK. A potential marker transcript for chronic pathologic conditions, such as diabetic nephropathy. Possible role in the response to persistent stress. J Biol Chem. 2000 Jun 9; 275(23):17838-47.

30 Kang SW, Adler SG, Lapage J, Natarajan R. p38 MAPK and MAPK kinase 3/6 mRNA and activities are increased in early diabetic glomeruli. Kidney Int. 2001 Aug;60(2):543-52.

31 Adhikary L, Chow F, Nikolic-Paterson DJ, Stambe C, Dowling J, Atkins RC, et al. Abnormal p38 mitogen-activated protein kinase signalling in human and experimental diabetic nephropathy. Diabetologia. 2004 Jul;47(7): 1210-22.
32 Sakai N, Wada T, Furuichi K, Iwata Y, Yoshimoto K, Kitagawa K, et al. Involvement of extracellular signal-regulated kinase and p38 in human diabetic nephropathy. Am J Kidney Dis. 2005 Jan; 45(1):54-65.

33 Bhattacharjee N, Barma S, Konwar N, Dewanjee S, Manna P. Mechanistic insight of diabetic nephropathy and its pharmacotherapeutic targets: an update. Eur J Pharmacol. 2016 Nov 15;791:8-24.

34 Wu J, Mei C, Vlassara H, Striker GE, Zheng F. Oxidative stress-induced JNK activation contributes to proinflammatory phenotype of aging diabetic mesangial cells. Am J Physiol Renal Physiol. 2009 Dec;297(6):F1622-31.

35 Huang F, Sheng XX, Zhang HJ. DUSP26 regulates podocyte oxidative stress and fibrosis in a mouse model with diabetic nephropathy through the mediation of ROS. Biochem Biophys Res Commun. 2019 Jul 30;515(3):410-6.

36 Sheng J, Li H, Dai Q, Lu C, Xu M, Zhang J, et al. DUSP1 recuses diabetic nephropathy via repressing JNK-Mff-mitochondrial fission pathways. J Cell Physiol. 2019 Mar;234(3): 3043-57.

37 Ge Y, Wang J, Wu D, Zhou Y, Qiu S, Chen J, et al. IncRNA NR_038323 suppresses renal fibrosis in diabetic nephropathy by targeting the miR-324-3p/DUSP1 axis. Mol Ther Nucleic Acids. 2019 Sep 6;17:741-53.

$38 \mathrm{Lu} \mathrm{C}$, Wu B, Liao Z, Xue M, Zou Z, Feng J, et al. DUSP1 overexpression attenuates renal tubular mitochondrial dysfunction by restoring Parkin-mediated mitophagy in diabetic nephropathy. Biochem Biophys Res Commun. 2021 Jun 25;559:141-7.

39 Huang H, Liu H, Tang J, Xu W, Gan H, Fan Q, et al. M2 macrophage-derived exosomal miR-25-3p improves high glucose-induced podocytes injury through activation autophagy via inhibiting DUSP1 expression. IUBMB life. 2020 Dec;72(12):2651-62.

40 Seccia TM, Caroccia B, Calò LA. Hypertensive nephropathy. Moving from classic to emerging pathogenetic mechanisms. J Hypertens. 2017 Feb;35(2):205-12.

41 Rodrigues-Díez R, Carvajal-González G, Sánchez-López E, Rodríguez-Vita J, Rodrigues Díez R, Selgas R, et al. Pharmacological modulation of epithelial mesenchymal transition caused by angiotensin II. Role of ROCK and MAPK pathways. Pharm Res. 2008 Oct; 25(10):2447-61.

42 Chen X, Cao Y, Wang Z, Zhang D, Tang W. Bioinformatic analysis reveals novel hub genes and pathways associated with hypertensive nephropathy. Nephrology. 2019 Nov; 24(11):1103-14.

43 Sousa-Lopes A, de Freitas RA, Carneiro FS, Nunes KP, Allahdadi KJ, Webb RC, et al. Angiotensin (1-7) inhibits Ang II-mediated ERK1/2 activation by stimulating MKP-1 activation in vascular smooth muscle cells. Int J Mol Cell Med. 2020;9(1):50-61.

44 Kucharska A, Rushworth LK, Staples C, Morrice NA, Keyse SM. Regulation of the inducible nuclear dual-specificity phosphatase
DUSP5 by ERK MAPK. Cell Signal. 2009 Dec; 21(12):1794-805.

45 Kidger AM, Rushworth LK, Stellzig J, Davidson J, Bryant CJ, Bayley C, et al. Dual-specificity phosphatase 5 controls the localized inhibition, propagation, and transforming potential of ERK signaling. Proc Natl Acad Sci U S A. 2017 Jan 17;114(3):E317-e26.

46 Zhang C, He X, Murphy SR, Zhang H, Wang $\mathrm{S}$, Ge Y, et al. Knockout of dual-specificity protein phosphatase 5 protects against hypertension-induced renal injury. J Pharmacol Exp Ther. 2019 Aug;370(2):206-17.

47 Earley S, Brayden JE. Transient receptor potential channels in the vasculature. Physiol Rev. 2015 Apr;95(2):645-90.

48 Thongnuanjan P, Soodvilai S, Chatsudthipong V, Soodvilai S. Fenofibrate reduces cisplatin-induced apoptosis of renal proximal tubular cells via inhibition of JNK and p38 pathways. J Toxicol Sci. 2016;41(3):339-49.

49 Jung YJ, Park W, Kang KP, Kim W. SIRT2 is involved in cisplatin-induced acute kidney injury through regulation of mitogen-activated protein kinase phosphatase-1. Nephrol Dial Transplant. 2020 Jul 1;35(7):1145-56.

$50 \mathrm{Xu}$ J, Ma L, Fu P. Eriocitrin attenuates ischemia reperfusion-induced oxidative stress and inflammation in rats with acute kidney injury by regulating the dual-specificity phosphatase 14 (DUSP14)-mediated Nrf2 and nuclear factor- $\kappa \mathrm{B}(\mathrm{NF}-\mathrm{\kappa B})$ pathways. Ann Transl Med. 2021 Feb;9(4):350.

51 Chang NJ, Weng WH, Chang KH, Liu EK, Chuang CK, Luo CC, et al. Genome-wide gene expression profiling of ischemia-reperfusion injury in rat kidney, intestine and skeletal muscle implicate a common involvement of MAPK signaling pathway. Mol Med Rep. 2015 May; 11(5):3786-93.

52 Stambe C, Nikolic-Paterson DJ, Hill PA, Dowling J, Atkins RC. p38 Mitogen-activated protein kinase activation and cell localization in human glomerulonephritis: correlation with renal injury. J Am Soc Nephrol. 2004 Feb;15(2):326-36.

53 Stambe C, Atkins RC, Tesch GH, Masaki T, Schreiner GF, Nikolic-Paterson DJ. The role of p38alpha mitogen-activated protein kinase activation in renal fibrosis. J Am Soc Nephrol. $2004 \mathrm{Feb} ; 15(2): 370-9$.

54 Ma FY, Flanc RS, Tesch GH, Han Y, Atkins RC, Bennett BL, et al. A pathogenic role for c-Jun amino-terminal kinase signaling in renal fibrosis and tubular cell apoptosis. J Am Soc Nephrol. 2007 Feb;18(2):472-84.

55 Li Z, Liu X, Tian F, Li J, Wang Q, Gu C. MKP2 inhibits TGF- $\beta 1$-induced epithelial-to-mesenchymal transition in renal tubular epithelial cells through a JNK-dependent pathway. Clin Sci. 2018 Nov 13;132(21):2339-55.

56 Wahab N, Cox D, Witherden A, Mason RM. Connective tissue growth factor (CTGF) promotes activated mesangial cell survival via upregulation of mitogen-activated protein kinase phosphatase-1 (MKP-1). Biochem J. 2007 Aug 15;406(1):131-8. 
57 Hou JH, Zhu HX, Zhou ML, Le WB, Zeng $\mathrm{CH}$, Liang SS, et al. Changes in the spectrum of kidney diseases: an analysis of 40,759 biopsy-proven cases from 2003 to 2014 in China. Kidney Dis. 2018 Feb;4(1):10-9.

58 Chuang HC, Chen YM, Hung WT, Li JP, Chen DY, Lan JL, et al. Downregulation of the phosphatase JKAP/DUSP22 in T cells as a potential new biomarker of systemic lupus erythematosus nephritis. Oncotarget. 2016 Sep 6; 7(36):57593-605.
59 Balada E, Felip L, Ordi-Ros J, Vilardell-Tarrés M. DUSP23 is over-expressed and linked to the expression of DNMTs in CD4(+) T cells from systemic lupus erythematosus patients. Clin Exp Immunol. 2017 Feb;187(2):242-50.

60 Jeong Y, Du R, Zhu X, Yin S, Wang J, Cui H, et al. Histone deacetylase isoforms regulate innate immune responses by deacetylating mitogen-activated protein kinase phosphatase-1. J Leukoc Biol. 2014 Apr;95(4):651-9.

61 Sallustio F, Serino G, Cox SN, Dalla Gassa A, Curci C, De Palma G, et al. Aberrantly methylated DNA regions lead to low activation of CD4+ T-cells in IgA nephropathy. Clin Sci. 2016 May;130(9):733-46.
62 Huang CY, Lin YC, Hsiao WY, Liao FH, Huang PY, Tan TH. DUSP4 deficiency enhances CD25 expression and CD4+ T-cell proliferation without impeding T-cell development. Eur J Immunol. 2012 Feb;42(2):47688.

63 Lu D, Liu L, Ji X, Gao Y, Chen X, Liu Y, et al. The phosphatase DUSP2 controls the activity of the transcription activator STAT3 and regulates TH17 differentiation. Nat Immunol. 2015 Dec;16(12):1263-73.

64 Chuang HC, Tan TH. MAP4K family kinases and DUSP family phosphatases in T-cell signaling and systemic lupus erythematosus. Cells. 2019 Nov 13;8(11):1433. 